\title{
Estimate of etched tracks by optical method and spark counting
}

\author{
Rajesh Kumar, K P Eappen*, A K Shukla, R M Tripathi and V D Puranik \\ Environmental Assessment Division, Bhabha Atomic Research Centre, Mumbai-400 085, India \\ E-mail : eappen@barc.gov.in
}

\begin{abstract}
Solid State Nuclear Track Detectors (SSNTD) are commonly used for long term measurements of radon, thoron and progeny concentrations. In the present study, we compared the optical counting method and the spark counting technique for counting the alpha tracks on LR 115 track detector films. The paper discusses the various parameters that are innate in the process. More than 300 films were counted by both optical microscope and spark counter and the results are compared. The overall results show that the tracks obtained by spark counting are marginally less compared to the optical measurement. A linear fit of the data gives a slope less that one, which indicates that both the methods are almost in good agreement for counting the tracks when the track density is low. At higher track densities the spark counter gives an underestimation with respect to actual number of tracks formed, which could be corrected using a relation obtained between the tracks measured by optical method and spark counting.
\end{abstract}

Keywords : LR 115, spark counter, radon.

PACS No. : $29.40 . \mathrm{Wk}$

\section{Introduction}

Passive radon monitoring devices based on alpha-particle etched track detectors are very attractive for the assessment of long-term radon exposures. The detector response is dependent on a number of factors related to the physical processes involved in the detection system. Primarily they fall upon to two processes viz. (i) the track development by chemical etching and (ii) track counting by optical or other conventional techniques. The potential of radon measurements with these detectors was recognized by Fleischer et al [1]. The first devices were proposed in 1967 and patented by Alter and Price [2] as Terradex TRACK ETCH, and in 1968 in a patent disclosure by Becker [3]. Latent tracks created by interaction of alpha particles in dielectric materials are developed by chemical etching and the tracks thus formed are counted. LR 115 track detector material being partially opaque and the tracks formed are through and through 
holes, optical transmission microscopes are the easiest and most widely used counting tool for track measurement. However, for large scale measurements using LR 115 films, the method of track counting using a spark counter introduced by Cross and Tommassino [4], is the most commonly used technique other than the optical system. In this case, the film with etched-through holes is placed between two electrodes forming a simple capacitor. As a dc voltage is applied to the capacitor, the track region starts conducting through the air column between the electrodes. An aluminized Mylar used as the top electrode vaporizes when the break down occurs, effectively removing the conductor on one side. In addition an RC circuit is used to remove the applied voltage at the time of sparking to avoid spark propagation in the neighboring holes. The linearity of the spark counter depends on a number of variables, including thickness of the aluminum layer of the electrode (Aluminized Mylar), RC circuit parameters, etc. In this study we carried out an experiment to find a comparison between the two methods of counting viz. the optical method and the spark counting.

\section{Experimental}

Underground uranium mine workers in Jaduguda are provided with personal dosimeters using LR 115 films for track detection. These dosimeters are brought back to the laboratory after stipulated exposure periods and processed the films using standard protocols for track development [5]. In the present study, dosimeters were selected from the various categories of employees, to cover the exposure ranges inside the mines, and processed the films for track developments and the etched track films were counted first by optical microscope followed by spark counting. More than 300 films were processed in this exercise. Tracks recorded by both the methods are presented in Figure 1.

\section{Error computation}

Figure 1 shows a good correlation among the two results but with a slope of 0.94

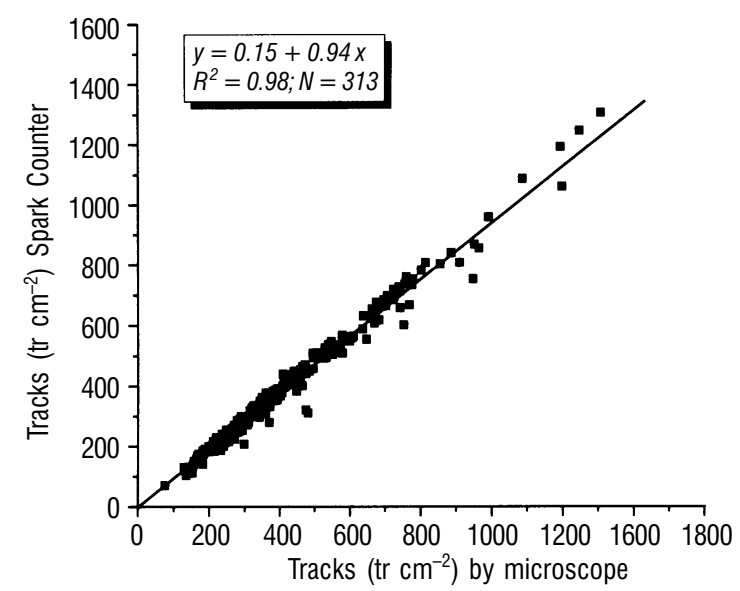

Figure 1. Comparison of tracks measured by microscope and spark counter. 
showing a trend of reduction in spark count values compared to optical counts. For a protocol maintained in a laboratory, parameters like etching conditions, aluminum thickness in Mylar film, spark counter parameters etc. can be taken as unity but, error propagation with track density is inherent in the method which is normally not reflected at low track densities.

At higher track count rates these differences are maximum because of the fact that the radius of the hole created on the aluminized mylar is more than one order of magnitude compared to the radius of the hole developed on the LR 115 film after etching. Assuming that the tracks observed by the optical method is true for the etched holes; percentage error with respect to spark counting is calculated from the tracks measured using spark counter and the microscope counting. Results are shown in Figure 2.

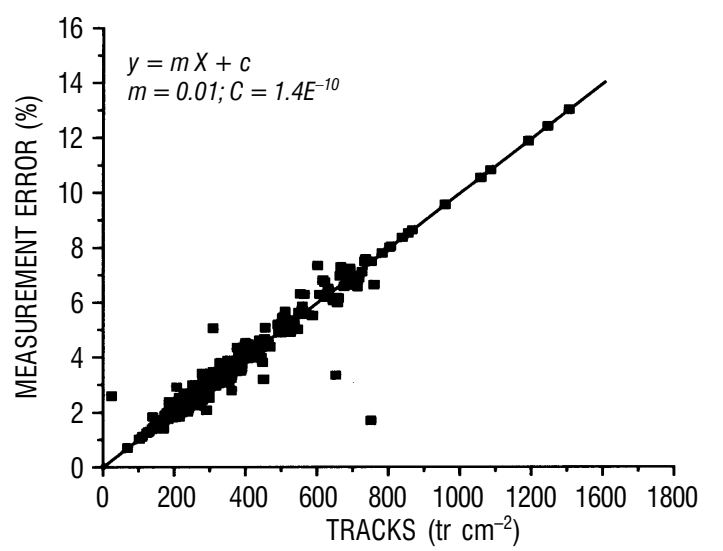

Figure 2. Measurement error with increase in track density.

It could be seen that the error propagates linearly and equation to the line in Figure 2 clearly indicates that the error is above 10 percentages when the track density is more than $1000 \mathrm{tr}$. $\mathrm{cm}^{-2}$. A correction to the counts obtained by spark counter can be made to obtain the true counts that are registered on the films.

\section{Correction to tracks by spark counting - a theoretical approach}

Let $r_{1}$ is the radius of the spark hole developed on the aluminum mylar film and $r_{2}$ is the radius of the latent track hole developed after etching, then the total number of tracks $\left(T_{T}\right)$ formed in LR 115 film can be equated to the tracks counted by the spark counter $\left(T_{s}\right)$ using eq. (1) [6].

$$
\begin{aligned}
& T_{T}=T_{S}\left[1+\frac{a \times T_{S}}{2}\right] \\
& a=\pi\left(r_{1}-r_{2}\right)^{2} ; \quad r_{1} \text { is the radius of the Al foil hole }>100 \mu \mathrm{m} \\
& r_{2} \text { is the radius of the track hole } \cong 6 \mu \mathrm{m}
\end{aligned}
$$


Since optical counting measures all through and through holes in the etched film, we consider the tracks $\left(T_{T}\right)$ obtained by optical counting are the actual number of tracks developed on the film. Following eq. (1), a power function fit of the data with $T_{S}$ on $X$-axis and $T_{T}$ on the $Y$-axis is done which is shown in Figure 3. Co-ordinate ' $A$ ' obtained through curve fitting of the data corresponds to ' $a / 2$ ' in eq. (1).

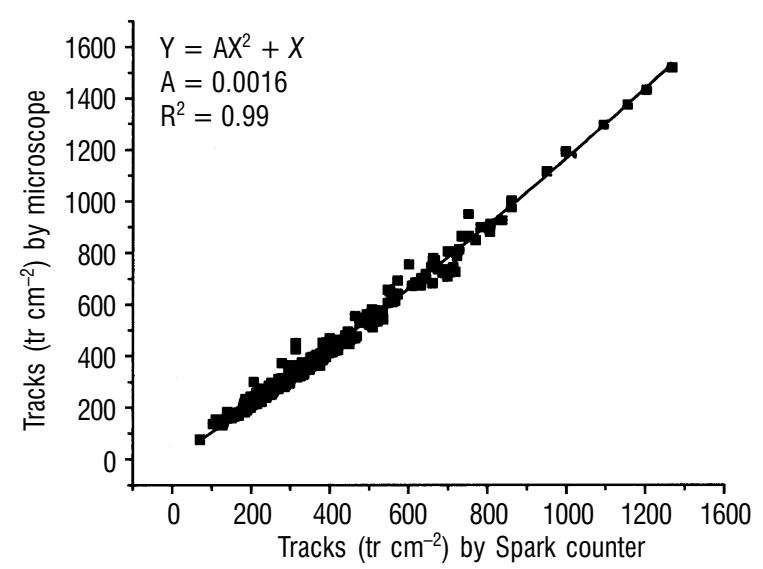

Figure 3. Relation between tracks by spark counting and microscope counting.

The value of $A$ in turn ' $a$ ' obtained from Figure 3 is used in eq. (1) to correct the tracks measured by spark counting to get the actual number of tracks registered on LR 115 films. A plot of tracks obtained by optical counting and the tracks after correction is shown in Figure 4.

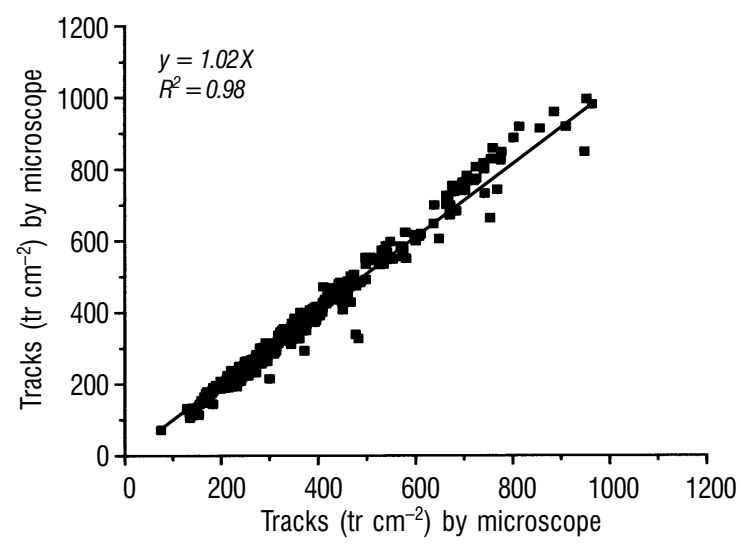

Figure 4. Comparison of tracks by microscope and spark counting (corrected).

\section{Results and discussion}

Results of counting by the two methods show that the tracks obtained by spark counting is an underestimation of the actual number of tracks registered on LR 115 films and the error is linearly propagated with increase in track density on films. Results from the measurements carried out on films exposed in underground uranium 
mines show clearly that the error is more than $10 \%$ above track density of 1000 tracks $\mathrm{cm}^{-2}$. As explained earlier, eq. (1) can be used to correct the tracks measured by spark counter to get the actual tracks recorded on LR 115 films. Figure 4 shows a very good correlation $\left(R^{2}=0.98\right)$ with a slope very close to one between the corrected spark count data and the microscope data, which clearly indicates that the method used to correct the tracks obtained from spark counting is proper in its approach.

Using the value of 'a' obtained from fitting the data (Figure 3), one can calculate $r_{1}$ and $r_{2}$, the radii of spark hole in mylar film and the latent track hole in LR 115 film respectively. In general terms, the diameter of the track opening, $D$, can be shown as :

$$
D=2 h \sqrt{\frac{V-1}{V+1}}
$$

where $V_{t} / V_{b}$ and $V_{t}$ and $V_{b}$ are etching rates $\left(\mu \mathrm{m} \mathrm{h} h^{-1}\right)$ for the track etch pit and bulk material of LR 115 film. In eq. (2) $V_{t}$ is taken as constant through out track etching, which may not be true in reality. Track etching rate, $V_{t}$ will depend on energy imparted per unit length of the material [7]. Hence, $V_{t}$ will be variable along the alpha track path with maximum at the end of the particle path. However, when $V_{t} \gg V_{b}$ for the case of alpha particles the change in difference is taken as minimal thereby eq. (2) is nearly valued. Hence, when $V>>1$, as is normally seen, eq. (2) will be simplified as :

$$
D \cong 2 h \text { and } h=V_{b} t
$$

From the protocol followed in our laboratory the bulk etch rate obtained is $4 \mu \mathrm{m} \mathrm{h}^{-1}$. We follow 90 minutes etching, thus diameter ' $D$ ' $\left(2 r_{2}\right)$ of the latent track hole developed in LR $115 \mathrm{film}$ is $12 \mu \mathrm{m}$. Knowing the value of $r_{2}$, the radius of the hole developed on the aluminized mylar can be calculated. Since $r_{1}$ will depend on various parameters like thickness of LR 115 film after etching, diameter of the etched track hole, RC circuitry of spark counter, density of aluminum on the mylar, sparking voltage etc., the value may vary between 100-200 $\mu \mathrm{m}$. Bhagwat and Soman [6] have reported a value of $86 \mu \mathrm{m}$ from their experiments. For $r_{2}$ equals to $12 \mu \mathrm{m}$ from the bulk etching rate of $4 \mu \mathrm{m} \mathrm{h}^{-1}$, the computed value of $r_{1}$ is $106 \mu \mathrm{m}$. A schematic representation of the holes developed both on LR 115 film as well as on the aluminized Mylar is shown in Figure 5.

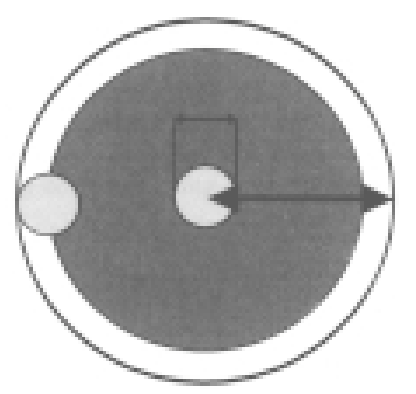

Figure 5. Diameters of holes created on LR 115 and Mylar Films. 


\section{Conclusions}

Spark count technique to measure etched tracks in LR 115 films is found to give an underestimation of the actual alpha tracks recorded on the films and the results show almost $10 \%$ variation at track density above 1000 tracks $\mathrm{cm}^{-2}$. Correction to the tracks is obtained through fitting parameter obtained from the tracks. The method of approach is found to give close proximity between the corrected tracks by spark counting and the tracks measured using microscope counting.

\section{References}

[1] R L Fleischer, P B Price and R M Walker Ann. Rev. Nucl. Sci. 151 (1965)

[2] H W Alter and P B Price US Patent 3665 (1972)

[3] K H Becker US Patent 3505 (1970)

[4] W G Cross and L Tommasino Radiat. Eff. 585 (1970)

[5] K P Eappen and Y S Mayya Radiat. Meas. 385 (2004)

[6] A M Bhagwar and S D Soman Ann. Conf. of Radiat. Prot. (1976)

[7] G Somogyi and A S Szalay Nucl. Instrum. Methods 10873 (1973) 